\title{
Comparative Direct Analysis of Type Ia Supernova Spectra. V. Insights from a Larger Sample and Quantitative Subclassification
}

\author{
David Branch, Leeann Chau Dang, \& E. Baron \\ Homer L. Dodge Department of Physics and Astronomy, University of Oklahoma, \\ Norman,OK; branch@nhn.ou.edu
}

\begin{abstract}
A comparative study of optical spectra of Type Ia supernovae (SNe Ia) is extended, in the light of new data. The discussion is framed in terms of the four groups defined in previous papers of this series: core normal $(\mathrm{CN})$; broad line (BL); cool (CL); and shallow silicon (SS). Emerging features of the SN Ia spectroscopic diversity include evidence (1) that extreme CL SN 1991bg-likes are not a physically distinct subgroup and (2) for the existence of a substantial number of SN 1999aa-like SSs that are very similar to each other and distinguishable from CN even as late as three weeks after maximum light. SN 1999aa-likes may be relatively numerous, yet not a physically distinct subgroup. The efficacy of quantitative spectroscopic subclassification of SNe Ia based on the equivalent widths of absorption features near $5750 \AA$ and $6100 \AA$ near maximum light is discussed. The absolute magnitude dispersion of a small sample of $\mathrm{CNs}$ is no larger than the characteristic absolute magnitude uncertainty.
\end{abstract}

Subject headings: supernovae: general

\section{INTRODUCTION}

This is the fifth in a series of papers on a comparative direct analysis of the optical spectra of Type Ia supernovae (SNe Ia). Papers I-IV (Branch et al. 2005, 2006, 2007, 2008) were concerned with a time series of spectra of the spectroscopically normal SN 1994D, and with samples of SN Ia spectra near maximum light, prior to maximum light, and after maximum light, respectively. In those papers, the parameterized supernova synthetic spectrum code SYNOW was used to study line identifications. Since those papers were written, the sample of spectra available to us has increased substantially, thanks especially to a large number of spectra published by Matheson et al. (2008). The purposes of this paper are to examine 
similarities, differences, and relationships among the spectra of the expanded sample, and to consider the efficacy of SN Ia quantitative spectroscopic subclassification based on the equivalent widths of absorption features near $5750 \AA$ and $6100 \AA$.

\section{THE CURRENT SAMPLE}

As in the previous papers, we confine our attention to optical spectra, from the Ca II $\mathrm{H}$ and $\mathrm{K}$ feature in the blue $(\sim 3700 \AA)$ to the Ca II infrared triplet (Ca II IR3) in the red $(\sim 9000 \AA)$. All spectra have been corrected for the redshifts of the host galaxies, and flattened according to the local normalization technique of Jeffery et al. (2007). Mild smoothing has been applied to some of the spectra. The current sample of 186 spectra of $65 \mathrm{SNe}$ Ia (Table 1) is restricted to the following ranges of epochs with respect to the time of maximum light in the B band: an early sample (day -11 or earlier); a 1 week premaximum sample (day -8 to day -6 ); a maximum light sample (day -3 to day +3 ); a 1 week postmaximum sample (day +6 to day +8 ); a 3 weeks postmaximum sample $($ day +19 to day +23$)$; and a 3 months postmaximum sample (day +80 to day +100 ). The only difference from previous papers of this series is that now, with more spectra available, the 1 week premaximum sample is from day -8 to day -6 , rather than from day -9 to day -5 as in Paper III.

\section{FOUR GROUPS}

In Paper II we divided the maximum light spectra of SNe Ia into four groups: core normal (CN), broad line (BL), cool (CL), and shallow silicon (SS). The group assignments were made on the basis of measurements of $W(5750)$ and $W(6100)$, the (pseudo) equivalent widths of absorption features near $5750 \AA$ and $6100 \AA$, as well as on the appearance (depth, width, and shape) of the $6100 \AA$ absorption. The $6100 \AA$ absorption is produced by the Si II $\lambda 6355$ transition and the $5750 \AA$ absorption usually is attributed to Si II $\lambda 5972$. A comparison of the $5750 \AA$ and $6100 \AA$ absorptions in maximum light spectra of the four groups is shown in Figure 1. The extreme BL SN 2006X has a very broad $6100 \AA$ absorption but only a weak $5750 \AA$ absorption; the $6100 \AA$ absorption of the extreme CL SN 1991bg is comparable to that of the CN SN 1998bu (except less blueshifted) but the $5750 \AA$ absorption is strong; and the extreme SS SN $1991 \mathrm{~T}$ has only a shallow $6100 \AA$ absorption and hardly any $5750 \AA$ absorption. (SN $1991 \mathrm{~T}$ would be less different from the others if a spectrum closer to maximum light was available.) In Papers II-IV the presentation and discussion were in terms of the four groups, although it was suggested that for the most part the 
spectra appeared to have a continuous distribution of properties, rather than breaking up into discrete subgroups - extreme SS SN 2002cx-likes being an apparent exception, and extreme CL SN 1991bg-likes being a possible exception.

In Paper II $W(5750)$ was plotted against $W(6100)$ for the 24 SNe of the maximum light sample. For the present paper all equivalent widths (Table 1) have been measured in a manner similar to that described by Hachinger et al. (2006). The flattening of the spectra has no significant effect on our measurements. Since each set of equivalent widths reported by us are for a specific spectrum obtained within 3 days of maximum light, while those reported by Hachinger et al. are based on multiple spectra obtained within 5 days of maximum light and extrapolated or interpolated to maximum light, exact agreement is not expected. For the $22 \mathrm{SNe}$ in both of our samples, the mean absolute differences in $W(5750)$ and $W(6100)$ are $2.7 \AA$ and $4.7 \AA$, respectively.

Figure 2 shows our current equivalent width plot, for 59 SNe. In general Figure 2 resembles its counterpart in Paper II, but now with more than double the data. The plot is most densely populated in the CN region, with members of the other groups more widely dispersed. For the present sample the domains of the four groups do not overlap, except for the maverick CL SN 1989B, which will be discussed in $\S 6$. Features that were not present in the plot of Paper II include (1) the presence of other CLs in the vicinity of SN 1986G, which had been rather isolated before, and (2) the clump of four SSs near $W(6100) \simeq 50 \AA$, $W(5750) \simeq 8 \AA$. These two developments will be discussed in $\S 6$ and $\S 7$.

Figure 3 is like Figure 2 but for the 28 SNe for which Hachinger et al. report $W(5750)$ and $W(6100)$ values $[E W(F 4)$ and $E W(F 5)$ in their notation], and with our group symbols. The distribution of SNe in Figure 3 is much like that of Figure 2 although with less data.

Figure 4 is like Figure 2, but also showing $W(5750)$ and $W(6100)$ values measured from synthetic spectra for the Si II ion, generated with the SYNOW code. (SYNOW is discussed in previous papers of this series. The parameter $v_{p h o t}$ is the velocity at the photosphere; $v_{e}$ is the e-folding velocity of the line optical depths; $v_{\max }$ is an optional maximum velocity of the line-forming region, and $T_{e x c}$ is the excitation temperature used to determine the relative optical depths of lines of an ion.) Several example Si II synthetic spectra are shown in Figure 5. The results in Figure 4 are generally consistent with how we fitted maximum light spectra in Paper II. We used $v_{e}=1000 \mathrm{~km} \mathrm{~s}^{-1}$ for CNs and larger values for BLs. For CLs we imposed maximum velocities on Si II, in order to saturate the $W(6100)$ values and obtain high values of $W(5750)$ by means of high line optical depth 1 . Overall, the lines in Figure 4

\footnotetext{
${ }^{1}$ Including the bluer component of the $5750 \AA$ blend in CLs (see Figure 1) provides a good empirical separation of CLs and BLs in Figure 2, but it should be noted that the bluer component is not accounted
} 
provide a good overview of the interpretation, at the SYNOW level, of the distribution of the SNe in this plot. However, the true nature of SN Ia spectra in the region of the $5750 \AA$ and $6100 \AA$ absorptions may be more complicated, in some cases involving S II, Fe II, and Fe III as well as Si II (Bongard et al. 2008). This may be why the positions of some SSs in Figure 4 are not accounted for by SYNOW spectra that contain only lines of Si II.2.

On the basis of five spectroscopic and photometric parameters other than $W(5750)$ and $W(6100)$, Benetti et al. (2005) assigned 26 SNe Ia to three groups: faint; high temporal velocity gradient (HVG); and low temporal velocity gradient (LVG). As discussed in Paper II, apart from a few borderline exceptions their faint group corresponds to our CLs; their HVG group corresponds to our BLs; and their LVG group includes both our CNs and SSs. Our understanding of the correspondence between our groups and those of Benetti et al., as discussed in Paper II, has not changed.

\section{CORE NORMALS}

The number of CNs has increased from 7 in Paper II to 15 now. The new ones are SN 1997dt, SN 1998V, SN 1998eg, and SN 2000fa (Matheson et al. 2008), SN 2003cg (EliasRosa et al. 2006), SN 2003du (Stanishev et al. 2007a), SN 2004S (Krisciunas et al. 2007), and SN 2005cf (Garavini et al. 2007; Wang et al. 2009).

By definition, at maximum light $\mathrm{CNs}$ have very similar spectra, except for differing strengths of high velocity (HV) Ca II features that form above 20,000 $\mathrm{km} \mathrm{s}^{-1}$. Among the new ones SN 2004S and SN 2005cf, like SN 2001el of Paper II, have strong HV Ca II at maximum light 3 . CNs are somewhat more diverse at 1 week premaximum and much more diverse at early times (Paper III).

At postmaximum epochs CNs remain impressively homogeneous. In Paper IV we attempted to illustrate the high degree of homogeneity of CNs at 3 months postmaximum by

for by Si II $\lambda 5972$ in SYNOW spectra (see, e.g., Figure 10 of Paper II, for SN 1991bg).

${ }^{2}$ Lines for very low $v_{e}$ values (very steep optical-depth gradients), $\lesssim 500 \mathrm{~km} \mathrm{~s}^{-1}$, fall farther to the left in Figure 4, near most of the SSs, but the synthetic line profiles are not good matches to the observed ones.

${ }^{3}$ A spectrum of Tycho Brahe's SN 1572 has been obtained by means of a light echo. Krause et al. (2008) showed that it is very similar to the time-integrated spectrum of the CN SN 2001el, and that of the CN SN 1994D except for the strong HV Ca II absorption of SN 1572 and SN 2001el. Thus Tycho's SN appears to have been a CN SN Ia with strong HV Ca II absorption. Krause et al. pointed out that by obtaining additional light-echo spectra it may be possible to construct a stereoscopic view of SN 1572, which would shed light on the geometry of the HV matter. 
plotting the logarithm of the flux with vertically displaced spectra. A better illustration of the homogenity is presented in Figure 6, which overplots the (non-logarithmic) flux spectra of the $6 \mathrm{CNs}$ of the 3 month postmaximum sample. Considering observational error and that the epochs of the spectra range from day +83 to day +91 , the homogeneity from $4000 \AA$ to $7000 \AA$ is remarkable and as argued in Paper IV is incompatible with the usual assumption that the spectra at these epochs (and even earlier) consist of optically thin emission lines. Instead, the blue part of the spectrum is dominated by resonance scattering features of permitted Fe II lines and the Na I feature. To the red of $7000 \AA$ the confusion is due to noise in the SN 1996X spectrum, to the differing strengths and amounts of deredshifting of the $7600 \AA$ terrestrial absorption, and to warping of the ends of some of the spectra by the local normalization process. Nevertheless, we cannot exclude that some of the differences are real, perhaps due to differing strengths of [Ca II] emission lines in this region of low permitted line opacity. To the red of the Ca II IR3 absorption near $8250 \AA$, the spectrum may consist mainly of optically thin emission lines, as usually assumed. However, the overall shape of spectra modelled with only optically thin forbidden lines will differ from the shape of spectra modelled with forbidden and permitted lines, because the optically thick permitted lines in the blue will transfer flux to the red.

\section{BROAD LINES}

The number of BLs has increased from 7 in Paper II to 20 now. The new ones are SN 1997do, SN 1998dh, SN 1998ec, SN 1999cc, SN 1999cl, SN 1999ej, SN 1999gd, SN 1999gh, and SN 2000B (Matheson et al. 2008), SN 2002dj (Pignata et al. 2008), SN 2004dt (Altavilla et al. 2007), and SN 2006X (Wang et al. 2008). Maximum light spectra are not available for SN 1997do, SN 1999gh, and SN 2000B, but it is clear from their 1 week postmaximum spectra that they should be classified as BL.

At maximum light BLs seem to have the same spectral features as CNs, but the $W(6100)$ values are higher. Some BLs are not very different from CN, so there is no reason to suppose a lack of continuity from CNs to these mild BLs. Extreme BLs have very high $W(6100)$ values but not high $W(5750)$ values, because (in SYNOW analyses) the Si II optical depth gradient is low ( $v_{e}$ is high) and the optical depth at the photosphere of $\lambda 6355$ is significant but not very high. This causes the $\lambda 6355$ absorption to extend over a wide velocity range, while the optical depth of the weaker $\lambda 5972$ transition is too low to produce significant absorption.

Although Figure 2 does not support any lack of continuity among BLs, it is clear that there is no single parameter sequence from CNs to extreme BLs. For example, the comparison 
in Figure 7 of the maximum light spectra of three extreme BLs (SN 2002bf, SN 2004dt, and SN 2006X) that are clustered at the lower right of Figure 2, shows that although the three have similar very broad $6100 \AA$ absorptions, SN 2004dt strongly differs from SN 2002bf and SN 2006X in several respects. The most obvious one is that SN 2004dt lacks strong absorption in the range $4800 \AA$ to $5100 \AA$, which in SYNOW analyses is attributed to HV Fe II (Paper II). SN 2002bf and SN 2006X do have strong HV Fe II absorption, with the corresponding emission partially filling in their S II absorptions and making them appear weaker than in SN 2004dt.

At 1 week premaximum, BLs are distinguishable from $\mathrm{CN}$, but at the earliest times the $\mathrm{CN}$ diversity is such that there is some overlap between $\mathrm{CN}$ and BL. At one week postmaximum, strong HV Fe II and partially filled in S II remains a distinguishing characteristic of most extreme BLs (see Figures 5 and 6 of Paper IV), but mild BLs are barely distinguishable from CN. By three weeks postmaximum, even extreme BLs are similar to CN.

\section{COOLS}

The number of CLs at maximum light has increased from 5 in Paper II to 11 now. The new ones are SN 1998bp, SN 1998de, SN 2000cn, and SN 2000dk (Matheson et al. 2008), SN 2004eo (Pastorello et al. 2007), and SN 2005bl (Taubenberger et al. 2008). A maximum light spectrum is not available for SN $2000 \mathrm{cn}$, but it is clear from its 1 week premaximum and 3 weeks postmaximum spectra that it should be classified as CL.

When Paper II was written, the sample of maximum light CLs consisted of SN 1991bg and two other extreme CLs, all with strong Ti II absorption; one less extreme CL, SN 1986G, with weaker Ti II; and one marginal CL, SN 1989B, with no identifiable Ti II absorption. Extreme SN 1991bg-like CLs have at times been regarded as a distinct SN Ia subgroup, but in Paper II we argued that there may be continuity from CNs through SN 1986G to SN 1991bg. It is interesting that among the five new maximum light CL spectra SN 2005bl is SN 1991bg-like, SN 1998de resembles SN 1986G, and three others (SN 1998bp, SN 2000dk, and SN 2004eo) are very similar to each other and similar to SN 1986G except for a lack of identifiable Ti II absorption. Figure 8 compares SN 1998bp, SN 2000dk, and SN 2004eo to SN 1986G. In LTE the Ti II strength increases rapidly with decreasing temperature (Hatano et al. 1999), so SN 1986G may just be slightly cooler than the others of Figure 8. Differences in titanium abundance may also be involved (Taubenberger et al. 2008). The existence of several SNe (SN 1998de, in addition to those of Figure 8) that resemble the intermediate CL SN 1986G strengthens the case for continuity (although probably not a single parameter sequence) from CN to extreme SN 1991bg-like CLs. 
In Figure 2 SN 1989B is in the domain of BLs. Nevertheless, in Paper II SN 1989B was included with CLs on the grounds that at maximum light its features are better resolved than those of BLs and that its $6100 \AA$ absorption is almost identical to that of the CL SN 1986G. The mild CL SN 2004eo now provides support for associating SN 1989B with CLs. Pastorello et al. (2007) refer to SN 2004eo as a transitional object, between the LVG, HVG, and FAINT groups of Benetti et al. (2005). In our classification scheme, SN 2004eo is a mild CL (Figure 8), and in many respects the spectra of SN 1989B resemble those of SN 2004eo; for example, at 1 week premaximum the spectra are very similar (Figure 9). The reason for the weaker $5750 \AA$ absorption of SN 1989B at maximum light is not known.

At 1 week premaximum CLs are distinguishable from CN. There are no CLs in the early sample. At 1 week and even 3 weeks postmaximum, CLs other than SN 1989B and SN 2004eo remain distinguishable from CN.

\section{SHALLOW SILICONS}

The number of SSs at maximum light has increased from 5 in Paper II to 20 now. The new ones are SN 1998ab, SN 1998es, SN 1999dq, SN 1999gp, and SN 2001V (Matheson et al. 2008), SN 1999ac (Garavini et al. 2005), SN 2000E (Valentini et al. 2003), SN 2003fg (Howell et al. 2006), SN 2005cg (Quimby et al. 2006), SN 2005hj (Quimby et al. 2007), SN 2005hk (Stanishev et al. 2007b), and SN 2006gz (Hicken et al. 2007). Maximum light spectra are not available for SN 1998ab and SN 2001V, but it is clear from their 1 week premaximum spectra that they should be classified as SS.

The SSs are a motley collection. At maximum light, among the new ones, SN 2005cg and SN 2005hj are not very far from CN; SN 1998ab is not far from the extreme SS SN 1991T; SN 1999ac is mildly peculiar in its own ways (Garavini et al. 2005; Phillips et al. 2006; Paper IV); SN 2003fg apparently has C II absorptions and is suspected of super-Chandrasekhar

mass ejection (Howell et al. 2006; Jeffery et al. 2008); SN 2006gz clearly has C II absorptions and may also be super-Chandrasekhar (Hicken et al. 2007); SN 2005hk is a near clone of SN 2002cx, both being quite distinct from typical SNe Ia (Li et al. 2003; Jha et al. 2006; Phillips et al. 2007; Stanishev et al. 2007b; Sahu et al. 2008). In general, SSs are distinguishable from $\mathrm{CN}$ at premaximum epochs and at 1 week postmaximum, but not at 3 weeks postmaximum.

Four new SSs not mentioned in the preceeding paragraph — SN 1998es, SN 1999dq, SN 1999gp, and SN 2001V are especially interesting not for their individuality but for their similarity to each other, and to SN 1999aa. This is illustrated in Figure 10 for the maximum 
light spectra of four of these (a maximum light spectrum is not available for SN 2001V).4 Figure 11 is similar but for four SNe at 1 week premaximum. (SN 1999gp does not have a spectrum at this epoch but SN 2001V does.) Figure 12 shows the 3 week postmaximum spectra of all five. When the SN 1999aa-likes of the 3 week postmaximum sample are compared to the well time-sampled series of SN 1994D spectra studied in Paper I, the SN 1999aa-likes are most like the day +14 spectrum of SN 1994D. The ratio of days past maximum light, 19 for SN 1999aa to 14 for SN 1994D is 1.36, the same as the ratio of the B-band light-curve stretch parameters of 1.143 for SN 1999aa and 0.838 for SN 1994D (Takanashi et al. 2008). The possibility that CNs and SSs age spectroscopically at the same rate that they decline photometrically will be explored in a separate paper.

\section{QUANTITATIVE SPECTRAL SUBCLASSIFICATION OF TYPE Ia SUPERNOVAE}

A quantitative subclassification of SN Ia spectra, preferably based on a single spectrum obtained near maximum light, would be useful to concisely convey a sense of what sort of SN Ia one is referring to. Before 1991, no spectroscopic subclassification scheme was used for SNe Ia, although it was known that some spectra differed from the norm (e.g., Phillips et al. 1987 on SN 1986G and Branch 1987 on SN 1984A). After the appearance of the "powerful" SN 1991T (Ruiz-Lapuente et al. 1992; Filippenko et al. 1992a; Phillips et al. 1992) and the "weak" SN 1991bg (Filippenko et al. 1992b; Leibundgut et al. 1993; Turatto et al. 1996), SNe Ia usually were said to be either normal, or peculiar SN 1991T-like, or peculiar SN 1991bg-like (e.g., Branch, Fisher, \& Nugent 1993). The term SN 1999aa-like was introduced by Li et al. (2001b) and recently we also have the enigmatic SN 2002cx-likes (Li et al. 2003; Jha et al. 2006). It has become clear (e.g., from Figure 2) that neither these few terms by themselves nor any single spectroscopic parameter can do justice to the multi-dimensional SN Ia maximum-light spectroscopic diversity.

We suggest that specifying the $W(5750)$ and $W(6100)$ parameters would be a useful quantitative spectroscopic subclassification. Only one spectrum near maximum light 5 is required, and an accurate redshift, sometimes not available for high redshift SNe Ia, is not

\footnotetext{
${ }^{4}$ Rest et al. (2008) obtained a light-echo spectrum of SNR 0509-67.5 in the Large Magellanic Cloud and showed that it is similar to the time-integrated spectra of SN 1998es, SN 1999aa, and SN 1999dq. Thus the LMC remnant appears to be that of a 1999aa-like SS.

${ }^{5}$ One week is a bit too far from maximum light because plots of $W(5750)$ and $W(6100)$ for the 1 week premaximum and 1 week postmaximum samples, although qualitatively like Figure 2, show a higher degree of overlapping domains than at maximum light.
} 
needed. (On the downside, some high redshift spectra do not reach restframe $6100 \AA$, and our present equivalent widths depend on a by-eye choice of where to begin and end the equivalent width integrations. A more precisely defined method such as that of Bongard et al. (2006) would be required for strictly reproducible results.) Specifying only the group name, without the equivalent widths, is not sufficient because, for example, it does not distinguish between mild BLs (which in the traditional sense would be termed "normal") and extreme BLs. Specifying the equivalent widths, without the group name, is insufficent because, for example, the CL SN 1989B falls within the domain of the BLs. Thus SN 2002bf could be denoted SN 2002bf (BL/11/175). This would concisely convey useful information to those familiar with the $W(5750)-W(6100)$ plane much as a spectral type of B2 IV concisely conveys useful information about Acrux.

\section{ABSOLUTE MAGNITUDES}

This series of papers has been confined almost entirely to optical spectra, but it is natural to wonder about the absolute magnitudes of the four spectroscopic groups. For our present sample the problem is that most host galaxies are not in the Hubble flow, which makes it difficult to compile a consistent set of distances. To estimate absolute magnitudes we have used data from Reindl et al. (2005) and Tammann, Sandage, \& Reindl (2008), including their estimates of host galaxy extinction. Distances in order of preference are based on (1) a Hubble constant of $65 \mathrm{~km} \mathrm{~s}^{-1} \mathrm{Mpc}^{-1}$ provided that host galaxy recession velocity 6 exceeds $2000 \mathrm{~km} \mathrm{~s}^{-1}$, (2) Cepheids and/or the tip of the red giant branch, and (3) membership in the Virgo, Fornax, or Ursa major clusters. The resulting Phillips relation (Phillips et al. 1999), for the SNe to which one of these distances can be assigned 7 , is shown in Figure 13. It is no surprise that on average $\mathrm{SSs}$ tend to be slowly declining (low $\Delta m_{15}$ ) and bright, and that CLs tend to be rapidly declining and faint. Figure 13 also shows that for this sample, BLs tend to have, on average, faster decline rates than CNs and they may contribute substantial scatter to the Phillips relation - perhaps not surprising in view of the considerable spectroscopic diversity among BLs. Most importantly, the spectroscopically homogeneous CNs may prove to be excellent standard candles: for this small sample of 9 CNs, $\bar{M}_{B}=-19.48, \sigma\left(M_{B}\right)=0.14$, with no apparent dependence of $M_{B}$ on $\Delta m_{15}$. The dispersion is no larger than the characteristic uncertainty in $M_{B}$. This is tantalizing, but the

\footnotetext{
${ }^{6}$ For SN 1999ej we have used $4690 \mathrm{~km} \mathrm{~s}^{-1}$, the mean velocity of the cluster in which it occurred (Jha, Riess, \& Kirshner, R. P. 2007).

${ }^{7}$ Owing to high host galaxy extinction, CN SN 1997dt, BL SN 1999cl, and CLs SN 1986G and SN 1989B are excluded.
} 
absolute-magnitude homogeneity of CNs must be tested with data on SNe in the Hubble flow, such as that forthcoming from the Supernova Factory (Bailey et al. 2008).

Figure 14 shows $M_{B}$ plotted against $W(5750)$. The sample is the same as in Figure 13 except that owing to the lack of a maximum light spectrum SN 1997br is missing. The 22 SNe having $W(5750)<22 \AA$ have $M_{B}=-19.50, \sigma\left(M_{B}\right)=0.17$. Evidently a maximum light spectrum, before a measurement of $\Delta m_{15}$ is possible, could eliminate much of the absolute magnitude scatter.

\section{SUMMARY}

By construction, core normals have a high degree of spectroscopic similarity. Extending away from CNs in Figure 2 are three "arms", which we call broad line, cool, and shallow silicon. These names are descriptive of the extreme members of the groups, but it is important to emphasize that the new data that have become available for this paper reinforce the impression that SNe Ia have a continuous distribution of spectroscopic properties rather than consisting of physically discrete subgroups. (SN 2002cx-likes remain an apparent exception. Valenti et al. 2009 suggest that they are core-collapse events, and should be classified as $\mathrm{SNe} \mathrm{Ib} / \mathrm{c}$.) Some BLs and SSs differ only mildly from CN and now, with several new CLs resembling the moderate CL SN 1986G, it seems likely that continuity also extends from CNs to extreme CLs. However, neither BLs nor SSs consist of single-parameter sequences extending from mild to extreme members, and the same appears to be true of CLs. It is not yet clear whether, apart from CNs, there is additional "clumping" in the spectroscopic properties; e.g., it will be interesting to see whether the apparent relatively high frequency of SN 1999aa-likes proves to be real or coincidental.

The quantitative spectroscopic subclassification suggested in $\S 8$ would be an improvement over anything in current use, but even that scheme cannot be expected to encompass the complex multi-dimensional spectroscopic diversity among SNe Ia. More observational data are needed to further illuminate it, and more work on explosion models is needed to understand it.

We are grateful to Rollin Thomas and Xiaofeng Wang for helpful comments and, as always, to all observers who provided spectra. This work has been supported by NSF grants AST 0506028 and AST 0707704, NASA LTSA grant NNG04GD36G, and DOE grant DEFG02-07ER41517. 


\section{REFERENCES}

Altavilla et al. 2007, A\&A, 475, 585

Bailey, S., et al. 2008, arXiv:astro-ph/0810.3499v2

Barbon, R., Iijima, T., \& Rosino, L. 1989, A\&A, 220, 83

Benetti, S., et al. 2004, MNRAS, 348, 261

Benetti, S., et al. 2005, ApJ, 623, 1011

Bongard, S., Baron, E., Smadja, G., Branch, D., \& Hauschildt, P. H. 2006, ApJ, 647, 513

Bongard, S., Baron, E., Smadja, G., Branch, D., \& Hauschildt, P. H. 2008, ApJ, 687, 456

Bowers, E., et al. 1997, MNRAS, 290, 663

Branch, D. 1987, ApJ, 316, L81

Branch, D., Baron, E., Hall, N., Melakayil, M., \& Parrent, J. 2005, PASP, 117, 545 (Paper I)

Branch, D., Fisher, A., \& Nugent, P. 1993, AJ, 106, 2383

Branch, D., et al. 1983, ApJ, 270, 123

Branch, D., et al. 2003, AJ, 126, 1489

—. 2006, PASP, 118, 560 (Paper II)

—. 2007, PASP, 119, 709 (Paper III)

—. 2008, PASP, 120, 135 (Paper IV)

Cristiani, S., et al. 1992, A\&A, 259, 63

Elias-Rosa, N., et al. 2006, MNRAS, 369, 1880

Filippenko, A. V. 1997, in Proc. NATO Advanced Study Institute, Thermonuclear Supernovae, ed. P. Ruiz-Lapuente, R. Canal, \& J. Isern (Dordrecht: Kluwer), 1

Filippenko, A. V., et al. 1992a, ApJ, 384, L15

—. 1992b, AJ, 104, 1543

Garavini, G., et al. 2004, AJ, 128, 387

—. 2005, AJ, 130, 2278

—. 2007, A\&A, 471, 527

Garnavich, P. M., et al. 2004, ApJ, 613, 1120

Gomez, G., Lopez, R., \& Sanchez, F. 1996, AJ, 112, 2094

Gomez, G., \& Lopez, R. 1998, AJ, 115, 1096 
Hachinger, S., Mazzali, P. A., \& Benetti, S. 2006, MNRAS, 370, 299

Hachinger, S., Mazzali, P. A., Tanaka, M., Hillebrandt, W., \& Benetti, S. 2008, MNRAS, 389,1087

Hamuy, M., et al. 2002, AJ, 124, 417

Hatano, K., Branch, D., Fisher, A., Millard, J., \& Baron, E. 1999, ApJS, 121, 233

Hernandez, M., et al. 2000, MNRAS, 319, 223

Hicken, M., Garnavich, P. M., Prieto, J. L., Blondin, S., DePoy, D. L., Kirshner, R. P., \& Parrent, J. 2007, ApJ, 669, 17

Howell, D. A., \& Nugent, P. 2004, in Cosmic Explosions in Three Dimensions, eds. P. Höflich, P. Kumar, \& J. C. Wheeler (Cambridge, CUP), p. 151

Howell, D. A., et al. 2006, Nature, 443, 308

Jeffery, D. J., Branch, D., \& Baron, E. 2008, ApJ, submitted; arXiv:astro-ph/0609804v3

Jeffery, D. J., Ketchum, W., Branch, D., Baron, E., Elmhamdi, A., \& Danziger, I. J. 2007, ApJS, 171, 493

Jha, S., Riess, A. G., \& Kirshner, R. P. 2007, ApJ, 659, 122

Jha, S., et al. 1999, ApJS, 125, 73

Jha, S., et al. 2006, AJ, 132, 189

Kirshner, R. P., et al. 1993, 415, 589

Kotak, R., et al. 2006, A\&A, 436, 1021

Krause, O., et al. 2008, Nature, 456, 617

Krisciunas, K., et al. 2007, AJ, 133, 58

Leibundgut, B., Kirshner, R. P., Filippenko, A. V., Shields, J. S, Foltz, C. B., Phillips, M. M., \& Sonneborn, S. 1991, ApJ, 371, L23

Leibundgut, B., et al. 1993, AJ, 105, 301

Leonard, D. C., Li, W., Filippenko, A. V., Foley, R. J., \& Chornock, R. 2005, ApJ, 632, 450

Li, W., Filippenko, A. V., Treffers, R. R., Riess, A. G., Hu, J., \& Qiu, Y. 2001b, ApJ, 546, 734

Li, W., et al. 1999, AJ, 117, 2709

—. 2001a, PASP, 113, 1178

—. 2003, PASP, 115, 453

Matheson, T., et al. 2008, AJ, 135, 1598 
Meikle, W. P. S., et al. 1996, MNRAS, 281, 263

Pastorello et al. 2007, MNRAS, 377, 1531

Patat, F., et al. 1996, MNRAS, 278, 111

Phillips, M. M., Lira, P., Suntzeff, N. B., Schommer, R. A., Hamuy, M., \& Maza, J. 1999, AJ, 118, 1766

Phillips, M. M., Wells, L. A., Suntzeff, N. B., Hamuy, M., Leibundgut, B., Kirshner, R. P., \& Foltz, C. B. 1992, AJ, 103, 1632

Phillips, M. M., et al. 1987, 99, 592

Phillips, M. M., et al. 2006, AJ, 131, 2615

Phillips, M. M., et al. 2007, PASP, 119, 360

Pignata, G., et al. 2008, MNRAS, 388, 971

Quimby, R., Höflich, P., \& Wheeler, J. C. 2007, ApJ, 666, 1083

Quimby, R., et al. 2006, ApJ, 636, 400

Reindl, B., Tammann, G. A., Sandage, A., \& Saha, A. 2005, ApJ, 624, 532

Rest, A., et al. 2008, ApJ, 680, 1137

Ruiz-Lapuente, P., Cappellaro, E., Turatto, M., Gouiffes, C., Danziger, I. J., Della Valle, M., \& Lucy, L. B. 1992, ApJ, 387, L33

Sahu, D. K. et al. 2008, ApJ, 680, 580

Salvo, M. E., Cappellaro, E., Mazzali, P. A., Benetti, S., Danziger, I. J., Pata, F., \& Turatto, M. 2001, MNRAS, 321, 254

Stanishev, V., et al. 2007a, A\&A, 469, 645

Stanishev, V., et al. 2007b, in The Multicolored Landscape of Compact Objects and Their Explosive Origins, AIP Conference Proceedings, 924, p. 336

Takanashi, N., Doi, M., \& Yasuda, N. 2008, MNRAS, 389, 1577

Tammann, G. A., Sandage, A., \& Reindl, B. 2008, Astr. Ap. Rev., 15, 289

Taubenberger, S., et al. 2008, MNRAS, 385, 75

Turatto, M., Piemonte, A., Benetti, S., Cappellaro, E., Mazzali, P. A., Danziger, I. J., \& Patat, F. 1998, ApJ, 116, 2431

Turatto, M., et al. 1996, MNRAS, 283, 1

Valenti, S., et al. 2009, Nature, submitted; arXiv:astro-ph/0901.2074v1

Valentini, G., et al. 2003, 595, 779 
Wang, L., et al. 2003, ApJ, 591, 1110

Wang, X., et al. 2008, ApJ, 675, 626

Wang, X., et al. 2009, ApJ, submitted; arXiv:astro-ph/0811.1205v1

Wells, L. A., et al. 1994, AJ, 108, 2233 


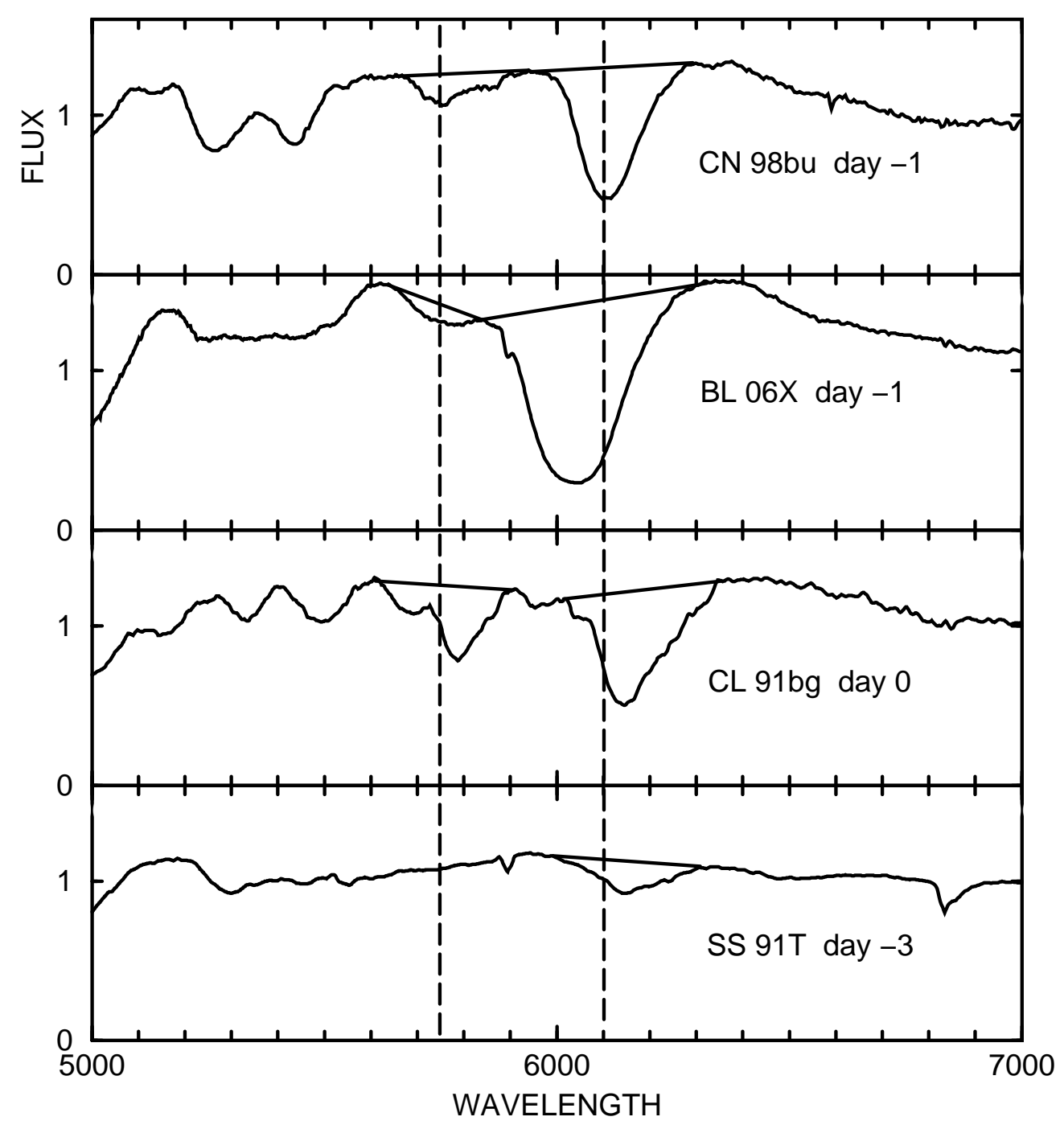

Fig. 1.- Comparison of maximum light spectra of the CN SN 1998bu, the extreme BL SN 2006X, the extreme CL SN 1991bg, and the extreme SS SN 1991T. Vertical dashed lines are at $5750 \AA$ and $6100 \AA$. Straight solid lines show how $W(5750)$ and $W(6100)$ were measured. (For clarity, the line for $W(5750)$ in SN $1991 \mathrm{~T}$ is not shown.) 


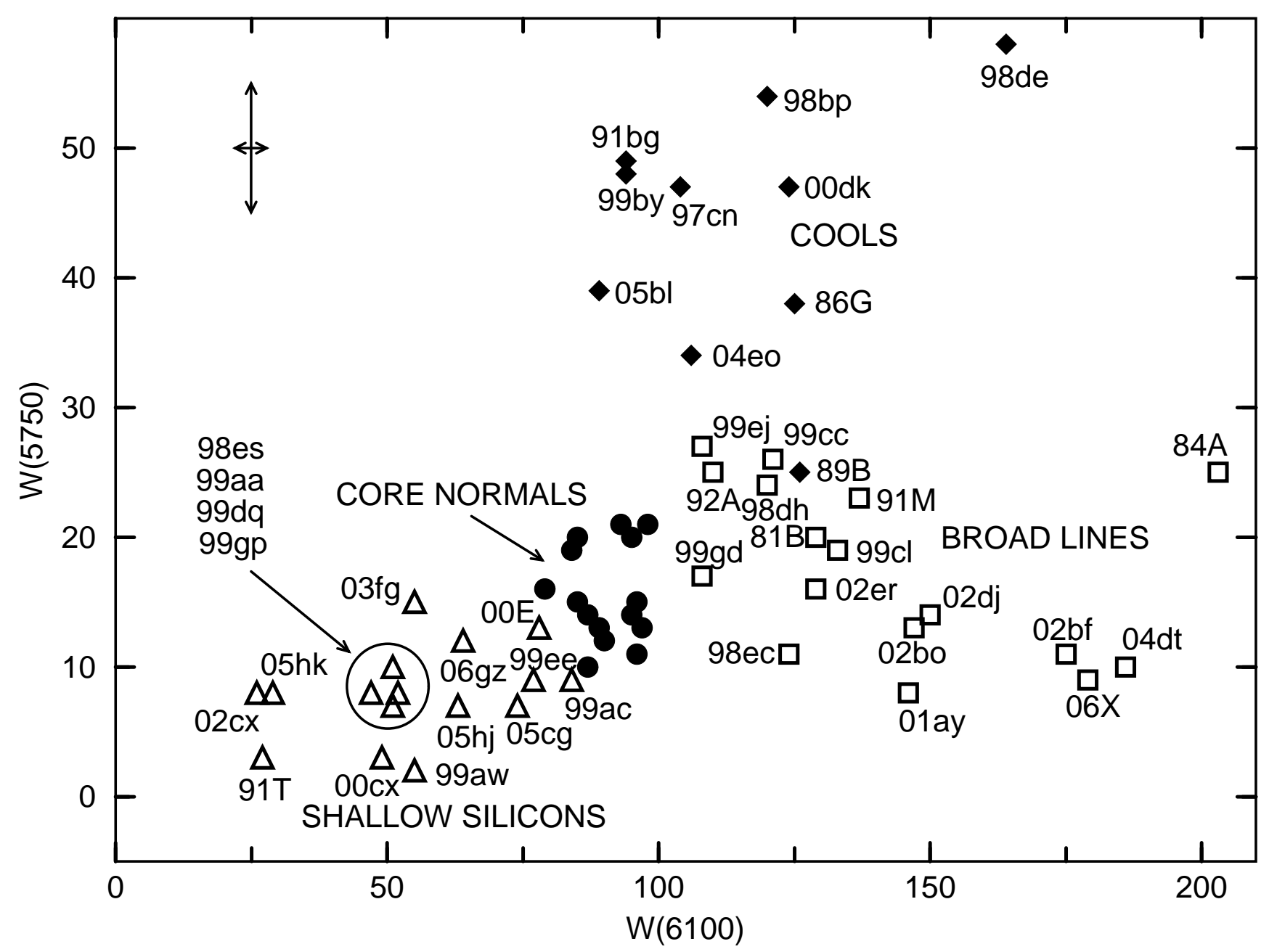

Fig. 2.- $W(5750)$ plotted against $W(6100)$. CNs are shown as filled circles, BLs as open squares, CLs as filled diamonds, and SSs as open triangles. Characteristic uncertainties of $3 \AA$ in $W(5750)$ and $5 \AA$ in $W(6100)$ are shown. For clarity individual CNs are not labeled. 


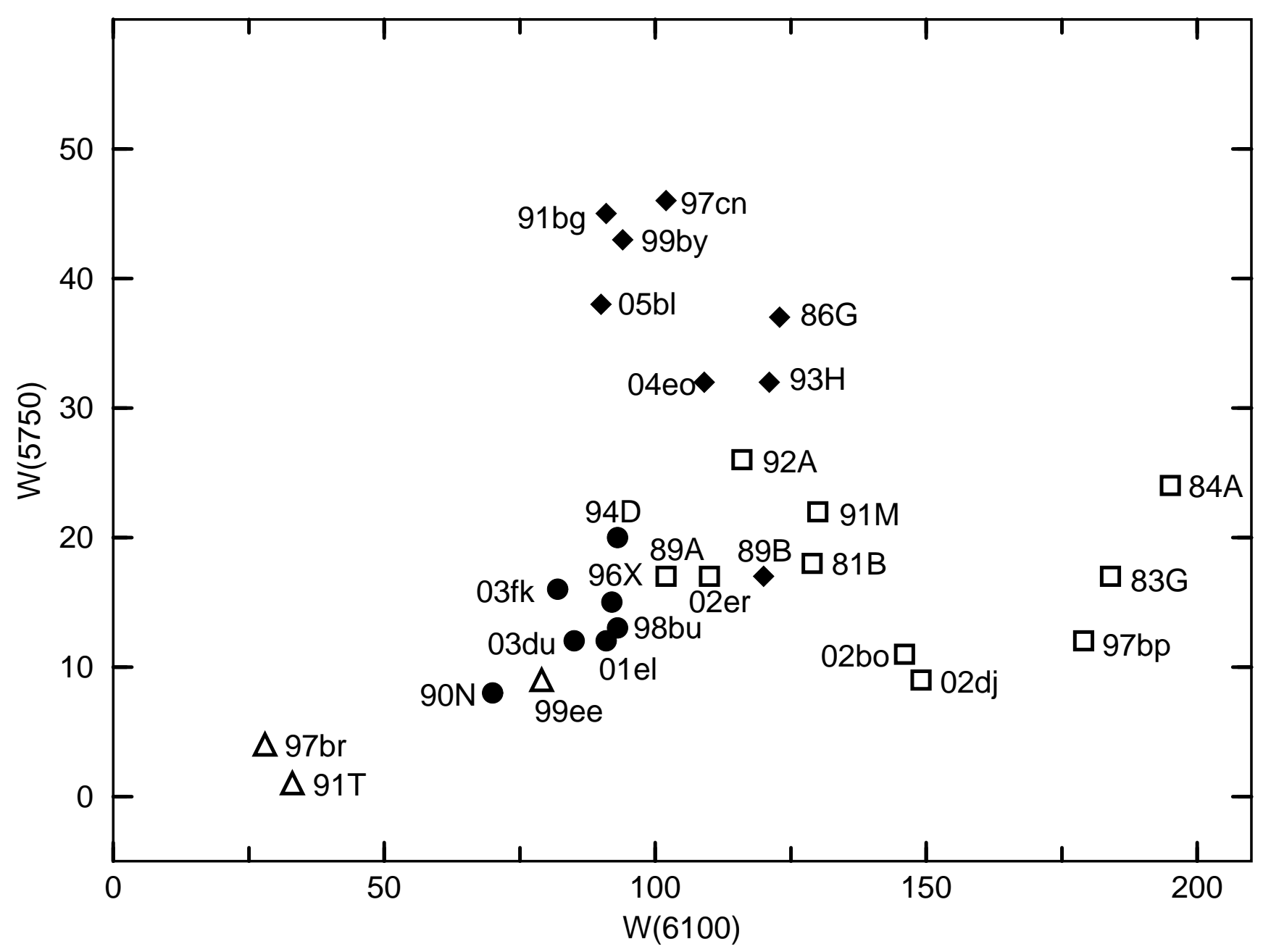

Fig. 3. - Like Figure 2 but for equivalent widths measured by Hachinger et al. 2006. 


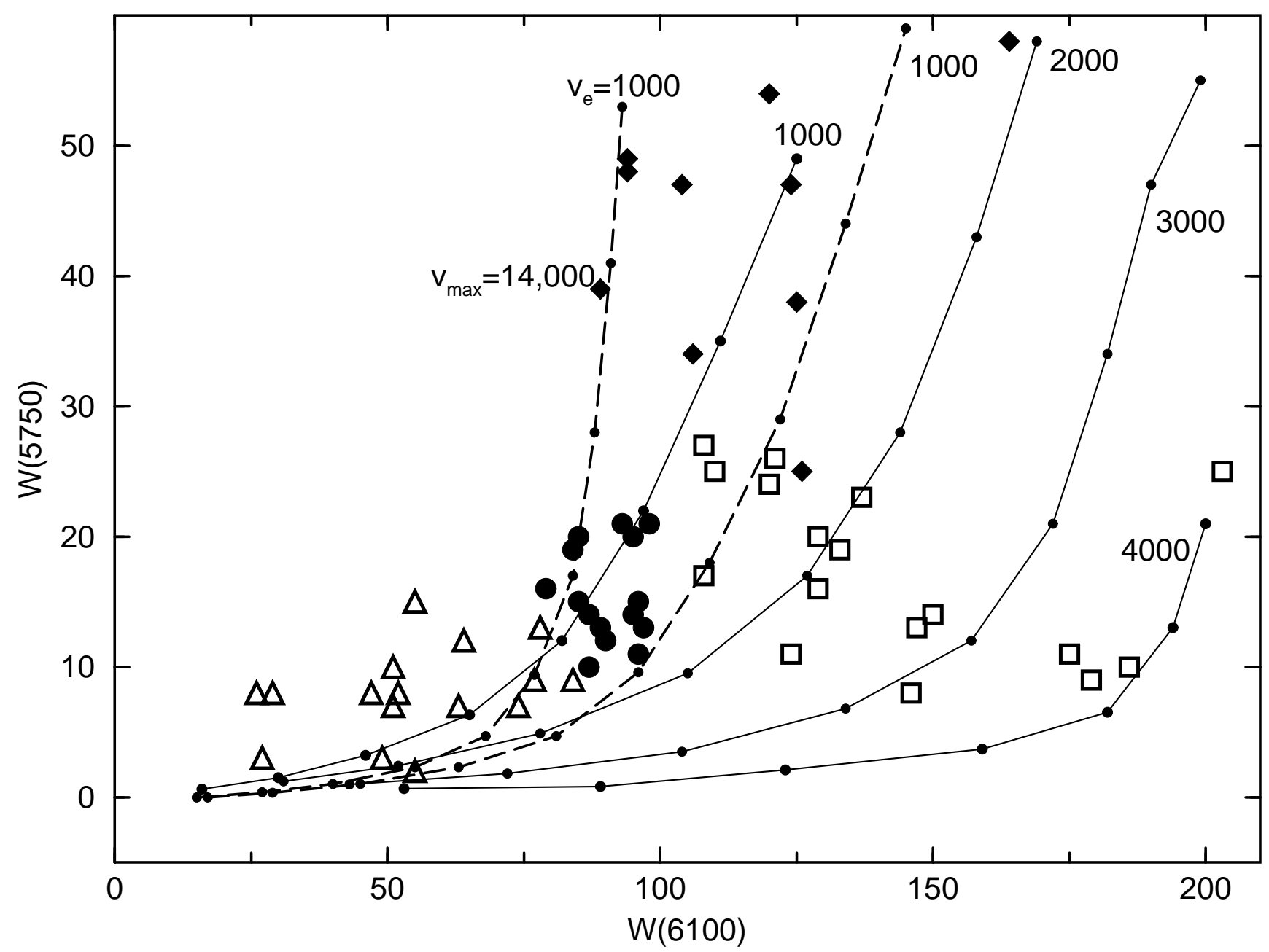

Fig. 4. - Like Figure 2 but with lines representing measurements of $W(5750)$ and $W(6100)$ from SYNOW synthetic spectra. Solid lines are for $v_{\text {phot }}=12,000 \mathrm{~km} \mathrm{~s}^{-1}, T_{\text {exc }}=10,000 \mathrm{~K}$, and $v_{e}$ from 1000 to $4000 \mathrm{~km} \mathrm{~s}^{-1}$ as indicated. Dashed lines (relevant only for CLs) are for $v_{\text {phot }}=11,000 \mathrm{~km} \mathrm{~s}^{-1}, T_{\text {exc }}=7000 \mathrm{~K}$, and $v_{e}=1000 \mathrm{~km} \mathrm{~s}^{-1}$. One of the dashed lines is for a maximum velocity $v_{\max }=14,000 \mathrm{~km} \mathrm{~s}^{-1}$, the other is for no maximum velocity. For each line, the optical depth at the photosphere of Si II $\lambda 6355$ begins at 0.5 (lower left point) and then increases by factors of 2 . 


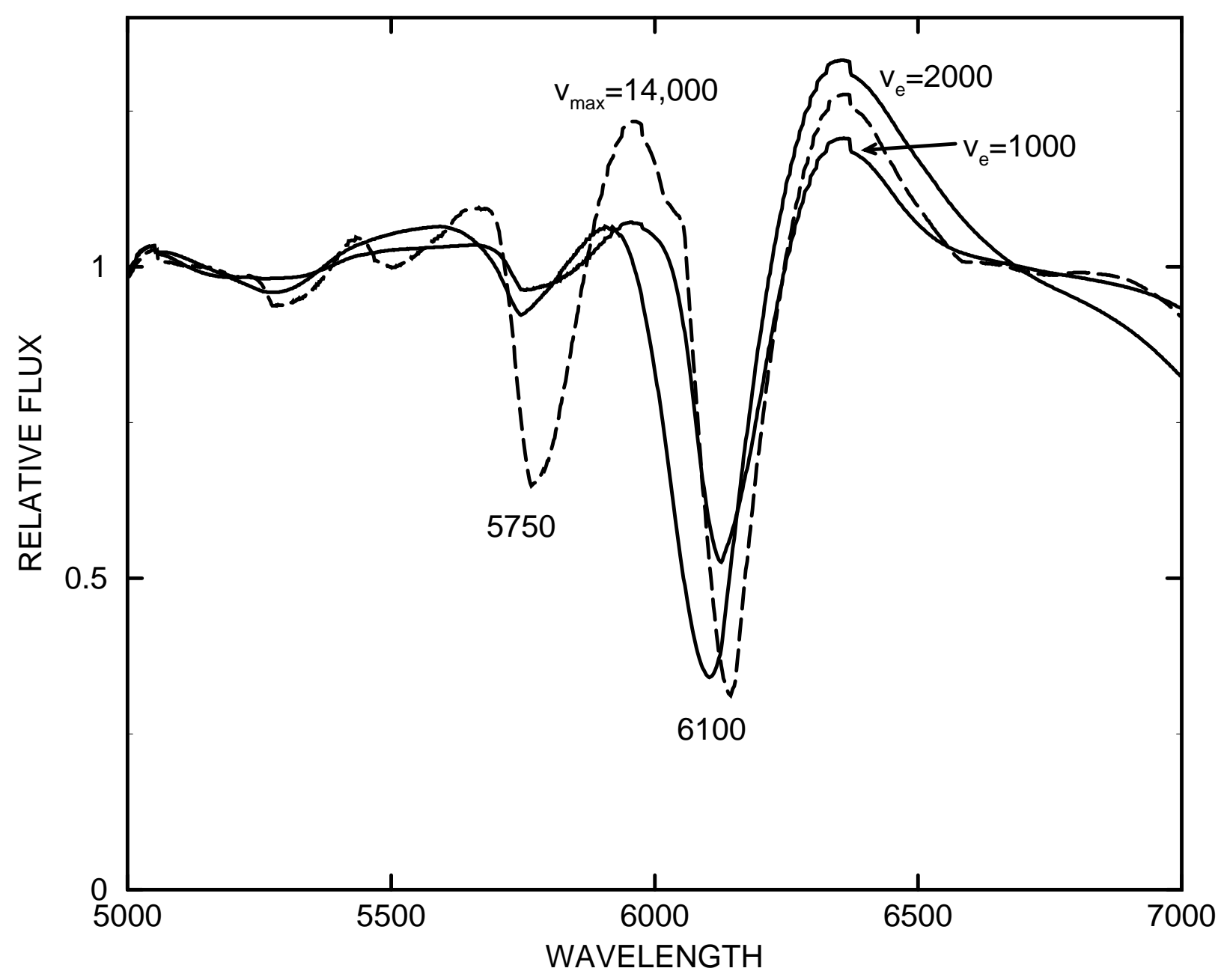

Fig. 5.- Example SYNOW synthetic spectra for Si II. Solid lines are for $v_{p h o t}=12,000$ $\mathrm{km} \mathrm{s}^{-1}, \tau=8, T_{e x c}=10,000 \mathrm{~K}$, and $v_{e}=1000$ and $2000 \mathrm{~km} \mathrm{~s}^{-1}$. Dashed line (relevant only to CLs) is for $v_{p h o t}=11,000 \mathrm{~km} \mathrm{~s}^{-1}, \tau=100, T_{e x c}=7000 \mathrm{~K}, v_{e}=1000 \mathrm{~km} \mathrm{~s}^{-1}$, and $v_{\max }=14,000 \mathrm{~km} \mathrm{~s}^{-1}$. The maximum velocity together with a high optical depth allows the $5750 \AA$ absorption to be strong without having the $6100 \AA$ absorption excessively strong. 


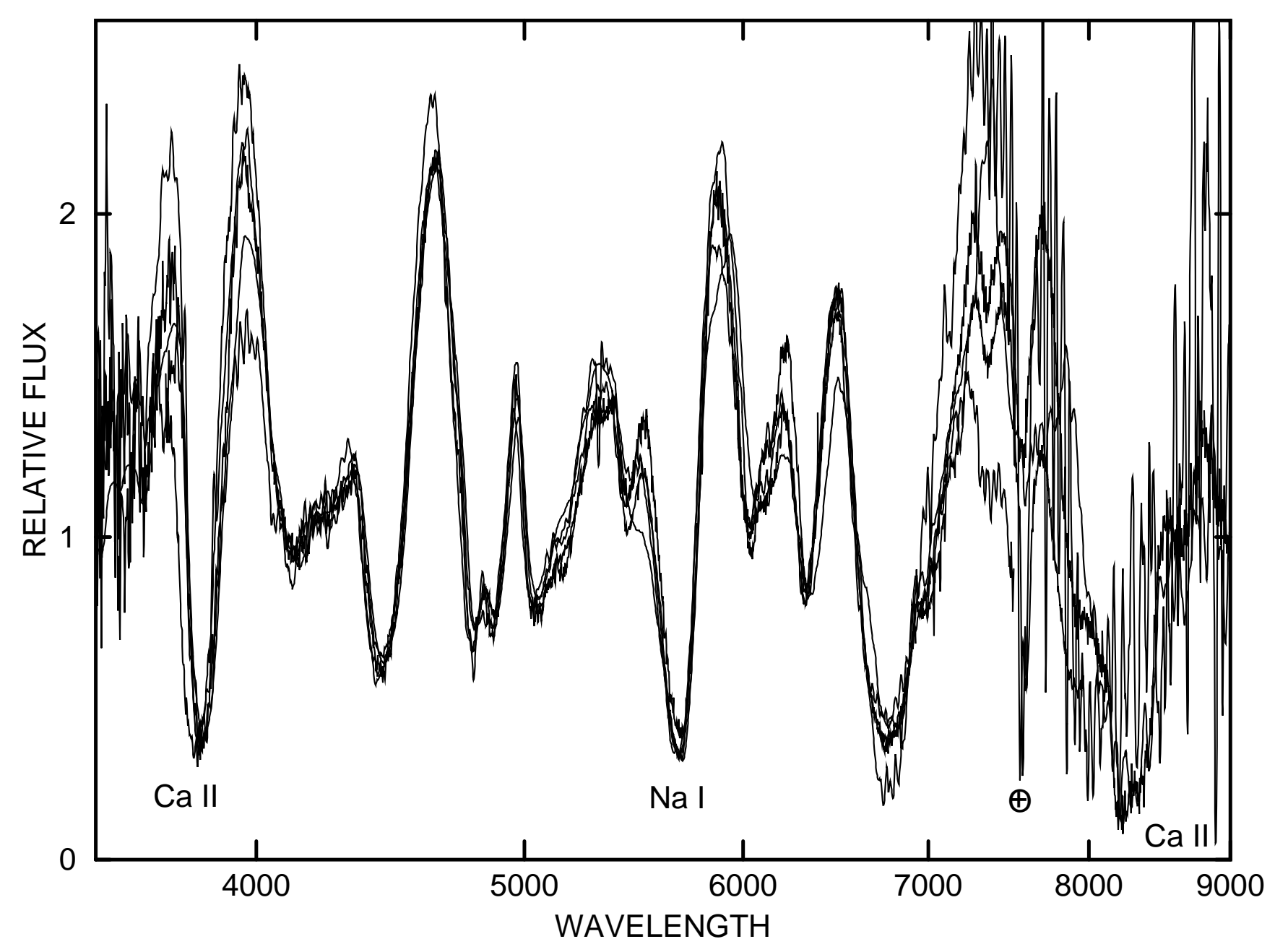

Fig. 6.- Spectra of the six CNs (SN 1994D, SN 1994ae, SN 1996X, SN 1998aq, SN 2003du, SN 2005cf) of the 3 months postmaximum sample. Unlabeled absorption features between $4000 \AA$ and $6500 \AA$ are attributed to permitted lines of Fe II (Paper IV). 


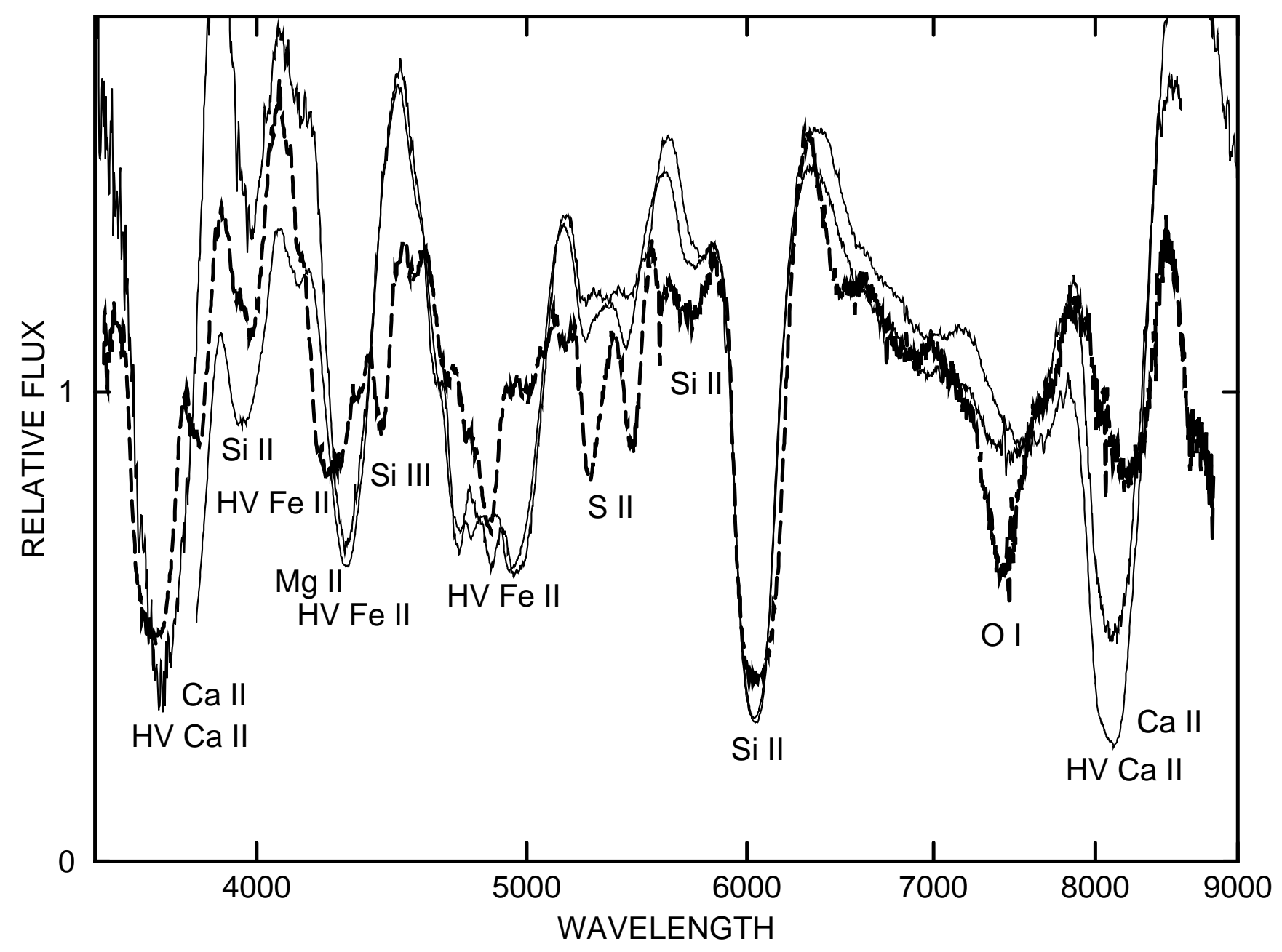

Fig. 7.- Maximum light spectra of 3 extreme BLs: SN 2004dt (dashed line), and SN 2002bf and SN 2006X (solid lines). The $6100 \AA$ absorptions are similar but SN 2004dt strongly differs from the other two in several respects. 


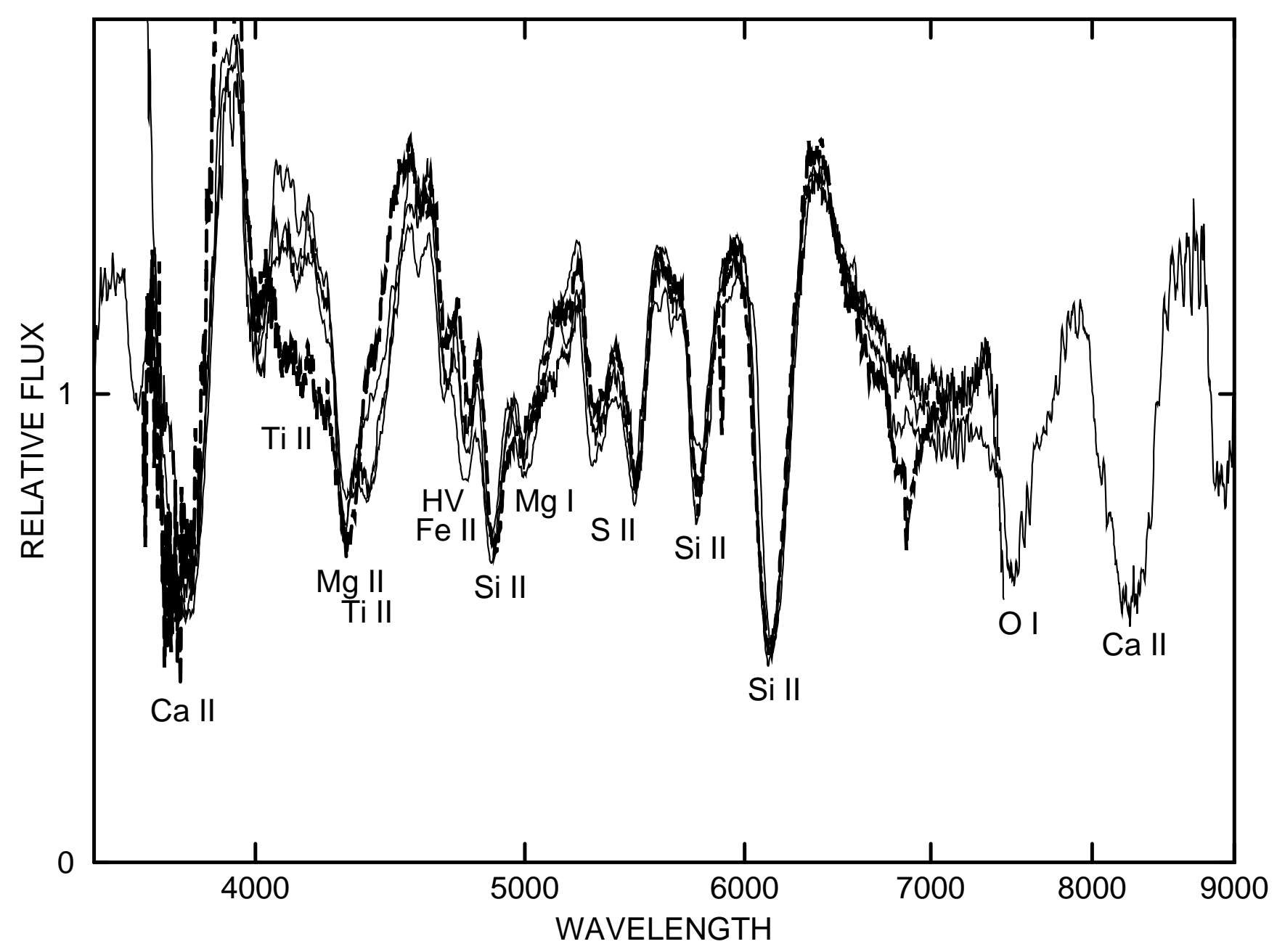

Fig. 8.- Maximum light spectra of 3 CLs (SN 1998bp, SN 2000dk, and SN 2004eo (solid lines), compared to SN 1986G (dashed line). Line identifications are for SN 1986G, which differs from the others in having stronger absorption near $4200 \AA$, attributed mainly to Ti II. 


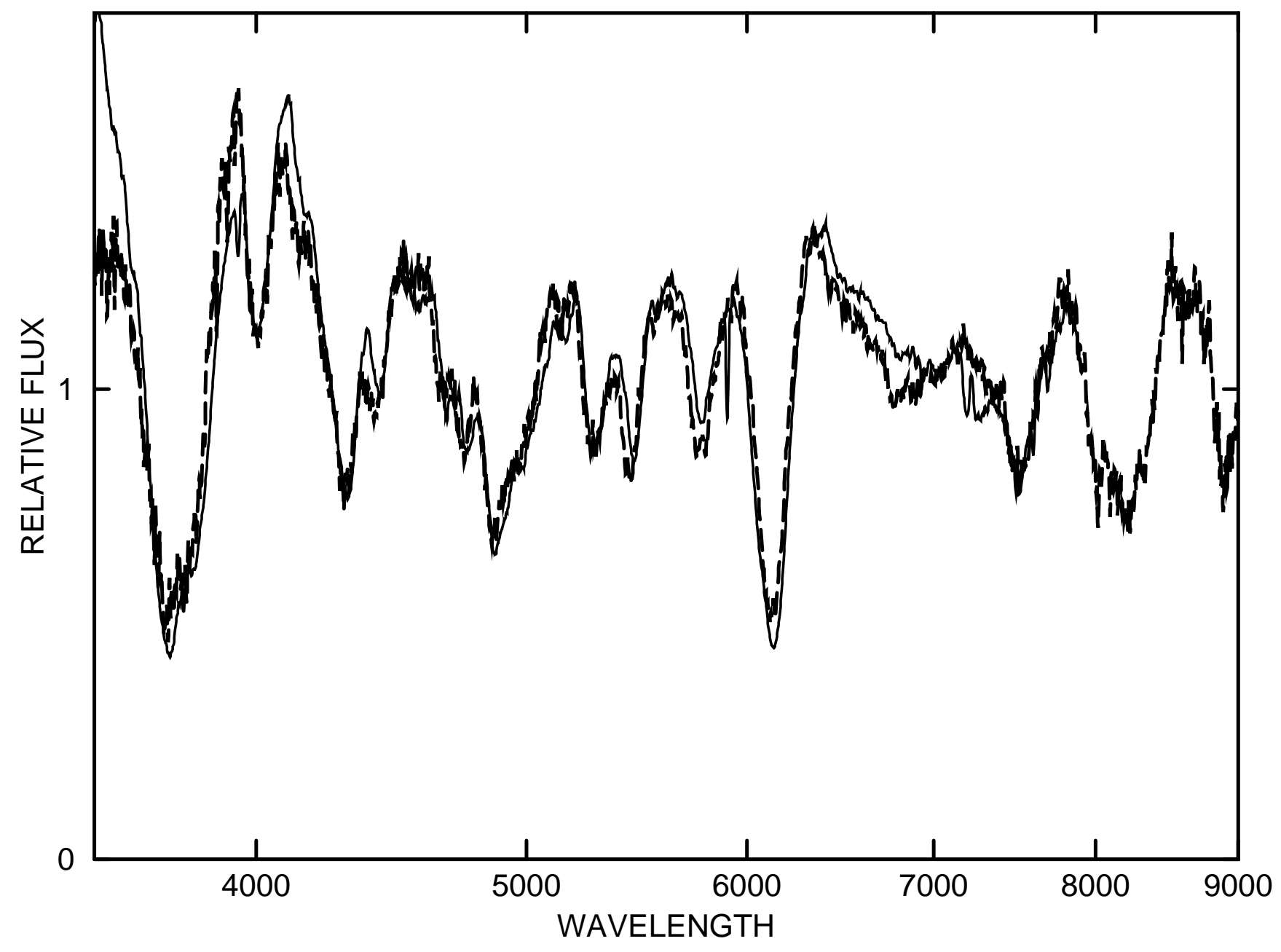

Fig. 9.- One week premaximum spectra of the mild CLs SN 1989B (solid line) and SN 2004eo (dashed line). The spectra are similar . 


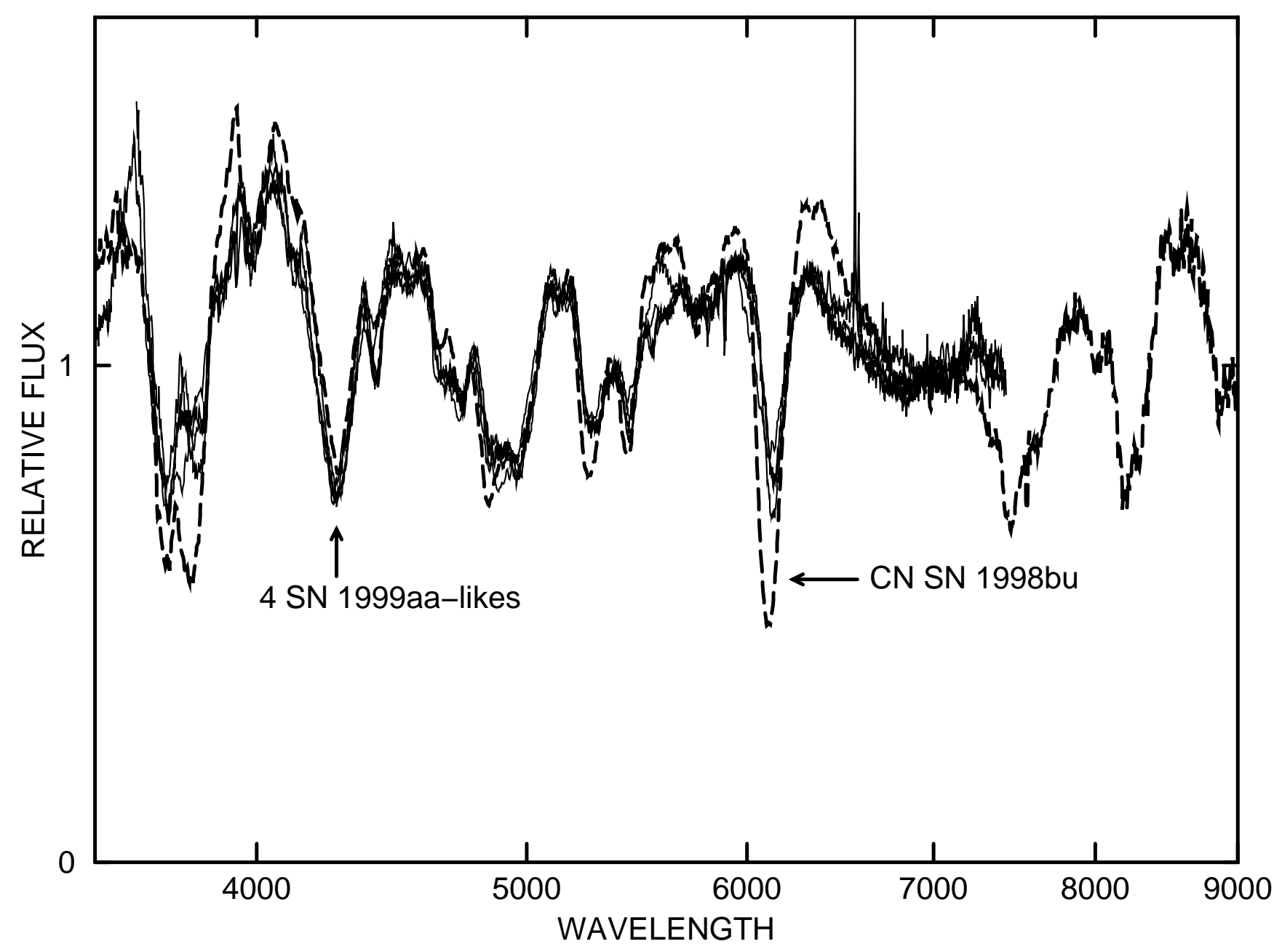

Fig. 10.- Maximum light spectra of 4 SSs - SN 1998es, SN 1999aa, SN 1999dq, and SN 1999gp (solid lines), compared to the CN SN 1998bu (dashed line). The 4 SN 1999aalikes are very similar to each other and distinguishable from SN 1998bu. 


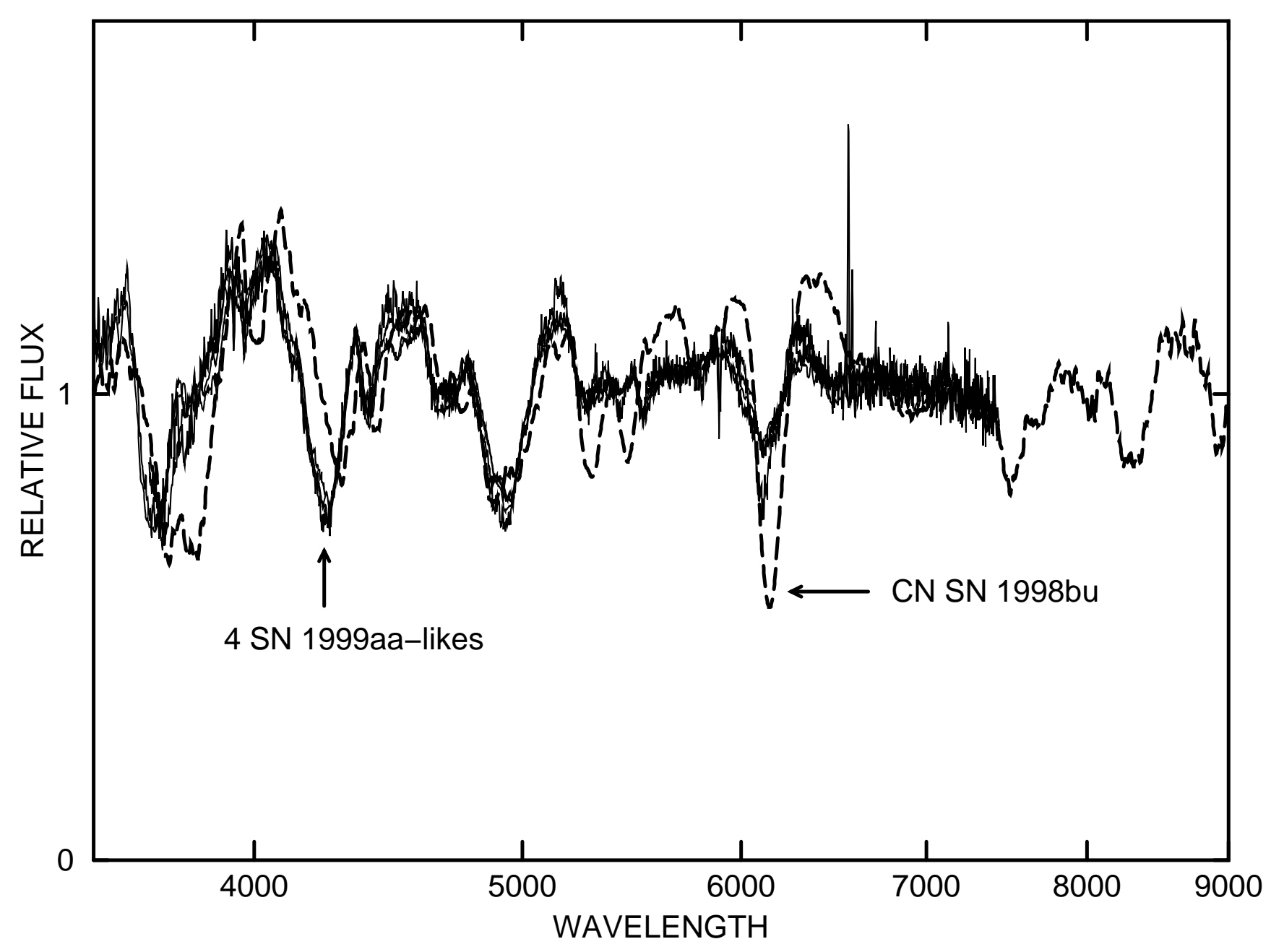

Fig. 11. - One week premaximum spectra of 4 SSs — SN 1998es, SN 1999aa, SN 1999dq, and SN 2001V (solid lines), compared to the CN SN 1998bu (dashed line). The 4 SN 1999aa-likes are very similar to each other (although SN 2001V has a mildly deeper $6100 \AA$ absorption than the others) and distinguishable from SN 1998bu. 


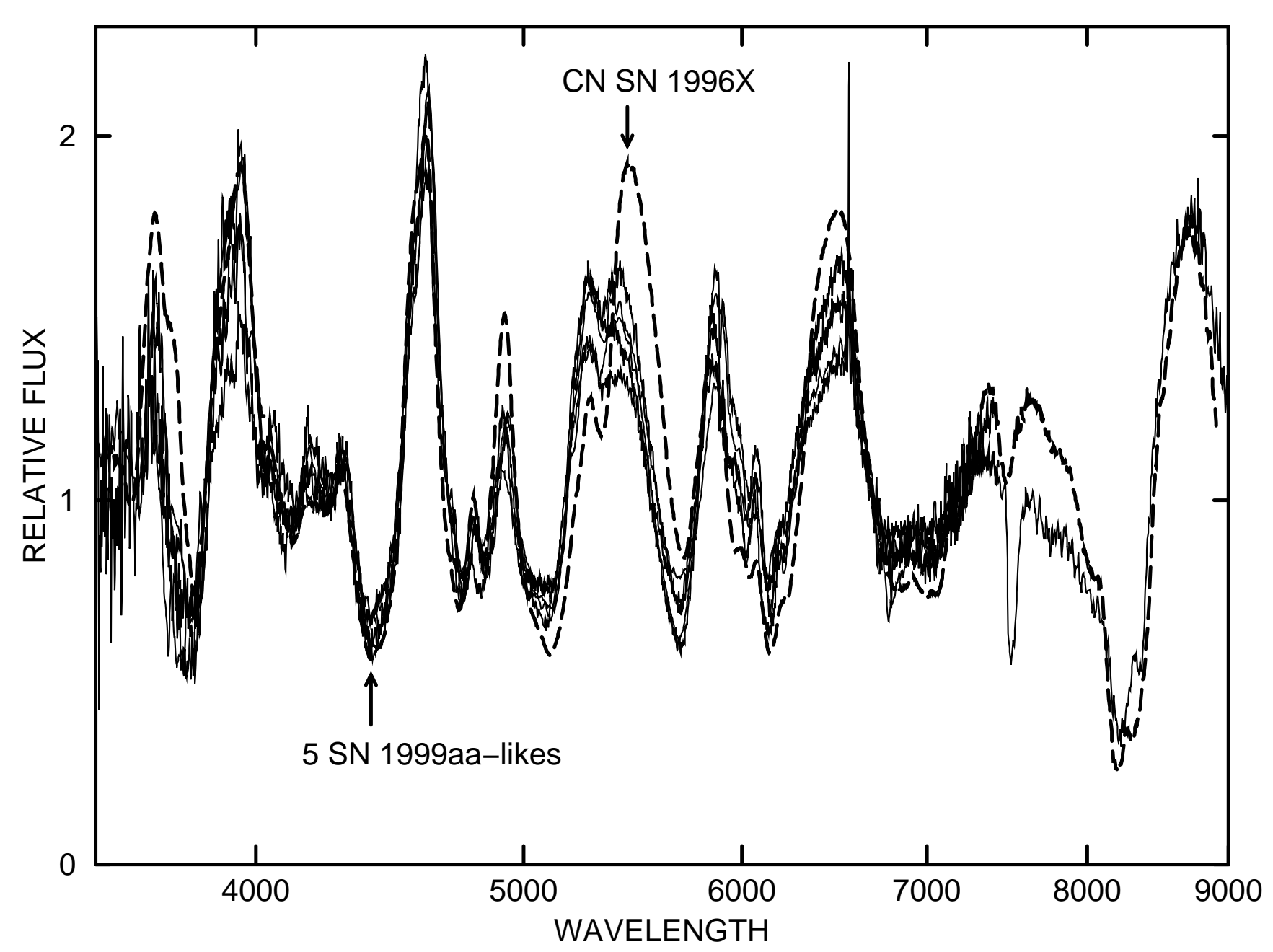

Fig. 12.- Three week postmaximum spectra of 5 SSs - SN 1998es, SN 1999aa, SN 1999dq, SN 1999gp, and SN 2001V (solid lines), compared to the CN SN 1996X (dashed line). The 5 SN 1999aa-likes are very similar to each other and distinguishable from SN 1996X. 


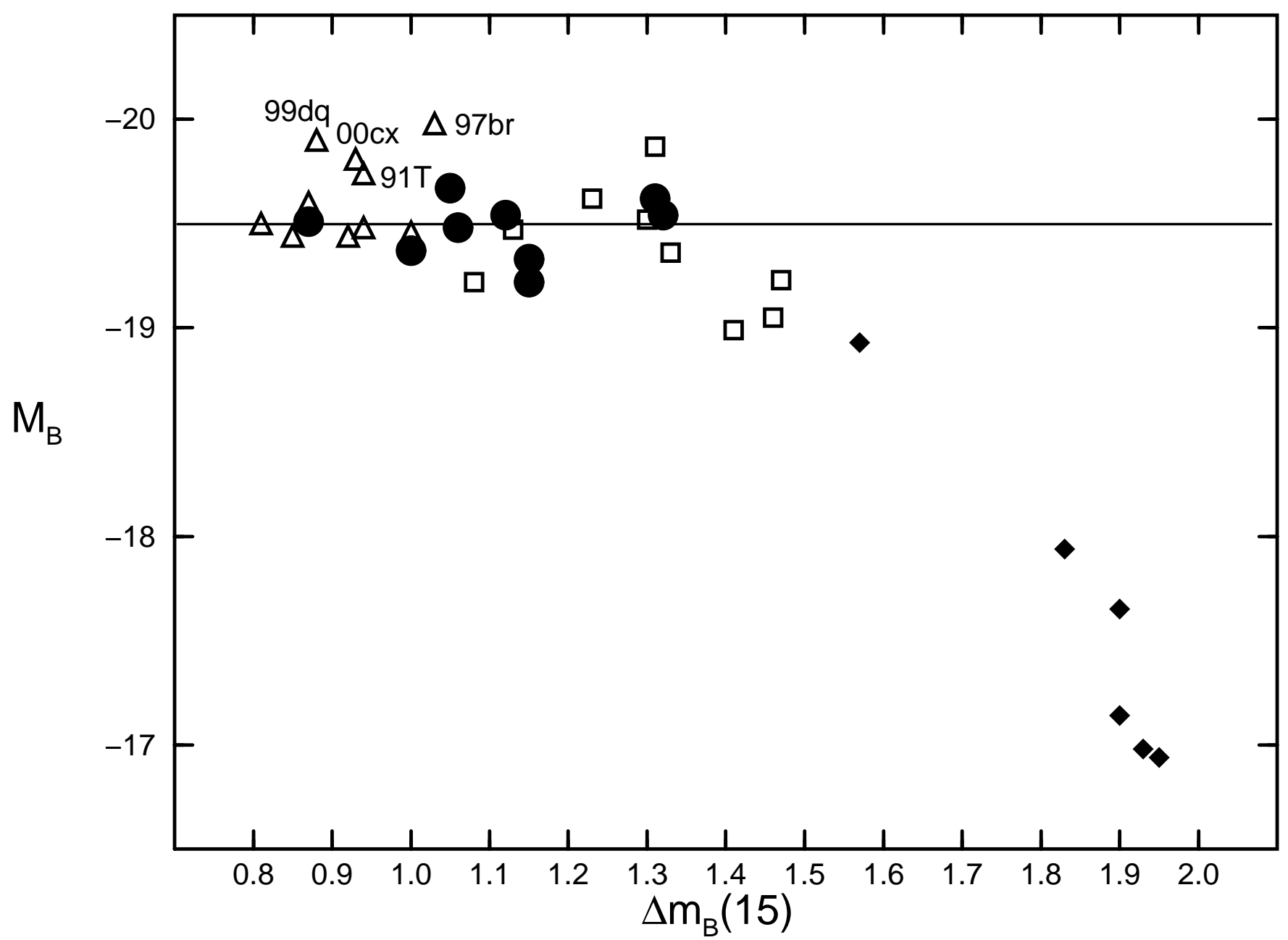

Fig. 13. - The Phillips relation. CNs are shown as filled circles, BLs as open squares, CLs as filled diamonds, and SSs as open triangles. The horizontal line is at $M_{B}=-19.5$. 


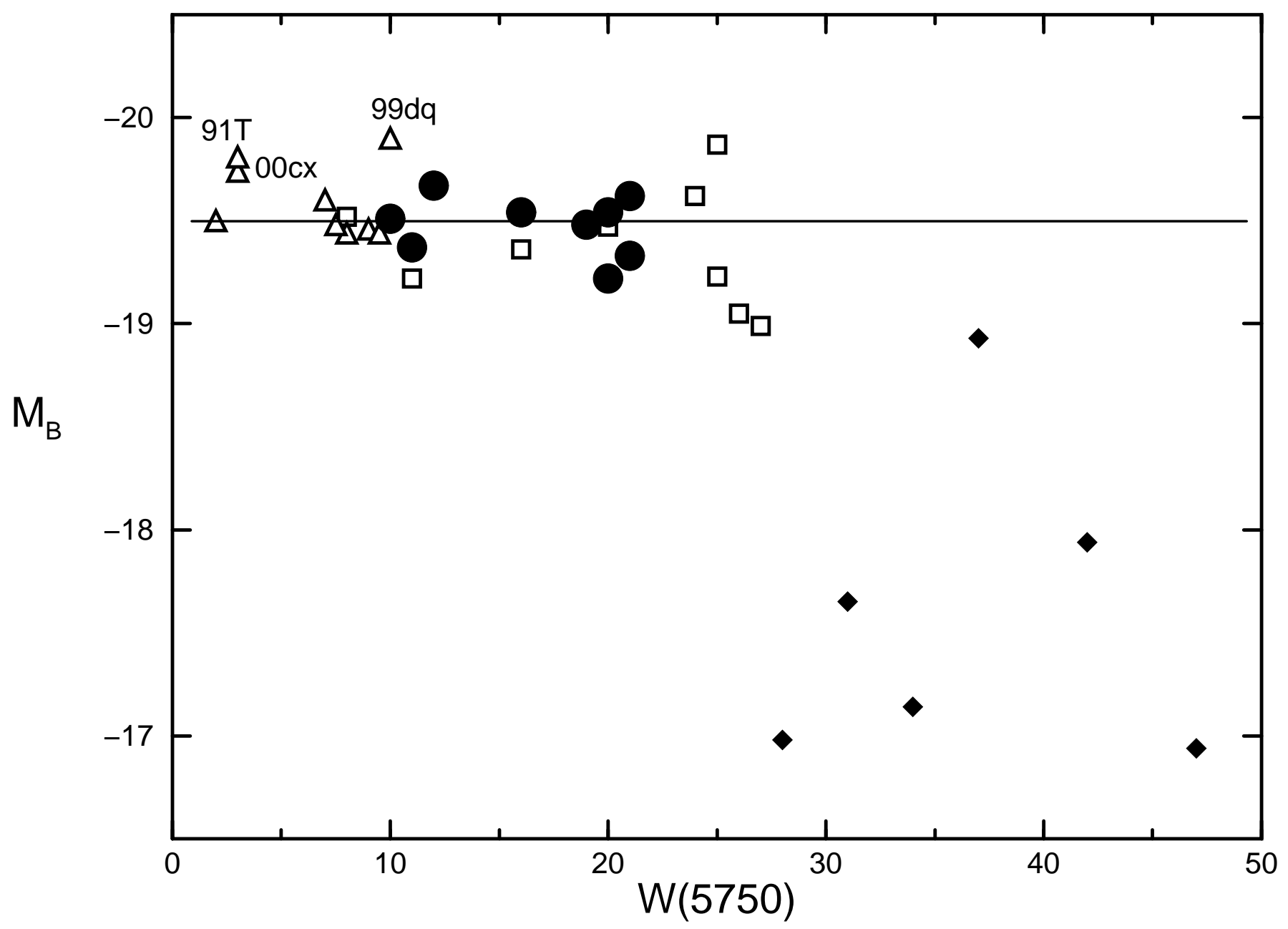

Fig. 14. $-M_{B}$ plotted against $W(5750)$. Symbols are as in Figure 13. The horizontal line is at $M_{B}=-19.5$. 
Table 1. The SN Ia Sample

\begin{tabular}{|c|c|c|c|c|c|}
\hline $\mathrm{SN}$ & Epochs (days) & Galaxy & $W(6100)(\AA)$ & $W(5750)(\AA)$ & References \\
\hline 1981B BL & $-2,22,93$ & NGC 4536 & 129 & 20 & Branch et al. 1983 \\
\hline 1984A BL & $-7,-3,8,19$ & NGC 4419 & 203 & 25 & Barbon et al. 1989 \\
\hline 1986G CL & $-6,-1,21,90$ & NGC 5128 & 125 & 38 & $\begin{array}{l}\text {-6: Phillips et al. } 1987 \\
\text {-1, 21, 90: Cristiani et al. } 1992\end{array}$ \\
\hline 1989B CL & $-7,0,8,19,92$ & NGC 3627 & 126 & 25 & Wells et al. 1994 \\
\hline $1990 \mathrm{~N} \mathrm{CN}$ & $-14,-8,-2,7,21$ & NGC 4639 & 90 & 12 & $\begin{array}{l}\text {-14, -8, 7: Leibundgut et al. } 1991 \\
\text {-2: Asiago SN Group } \\
\text { 21: Filippenko et al. } 1992 \mathrm{~b}\end{array}$ \\
\hline 1991M BL & 3,81 & IC 1151 & 137 & 23 & $\begin{array}{l}\text { 3: Gomez et al. } 1996 \\
\text { 81: Gomez \& Lopez } 1998 \text { లే }\end{array}$ \\
\hline 1991T SS & $-11,-7,-3,83$ & NGC 4527 & 27 & 3 & $\begin{array}{l}-11,-7,-3: \text { Phillips et al. } 1992 \\
\text { 83: A. V. Filippenko, unpublished }\end{array}$ \\
\hline 1991bg CL & $0,19,91$ & NGC 4374 & 94 & 49 & Filippenko et al. $1992 \mathrm{a}$ \\
\hline 1992A BL & $-6,-1,6$ & NGC 1380 & 110 & 25 & $\begin{array}{l}-6,-1: \text { P. Challis, unpublished } \\
\text { 6: Kirshner et al. } 1993\end{array}$ \\
\hline 1994D CN & $-12,-8,-1,7,19,87$ & NGC 4526 & 98 & 21 & $\begin{array}{l}-12,-8,-1: \text { Meikle et al. } 1996 \\
\text { 7: Patat et al. } 1996 \\
\text { 87: Filippenko } 1997\end{array}$ \\
\hline 1994ae CN & 0,89 & NGC 3370 & 87 & 10 & $\begin{array}{l}\text { 0: Howell \& Nugent } 2004 \\
89 \text { Bowers et al. } 1997\end{array}$ \\
\hline 1996X CN & $-2,7,22,87$ & NGC 5061 & 85 & 20 & Salvo et al. 2001 \\
\hline 1997br SS & $-7,8$ & ESO 576-G40 & $\ldots$ & $\ldots$ & Li et al. 1999 \\
\hline 1997cn CL & 3 & NGC 5490 & 104 & 47 & Turatto et al. 1998 \\
\hline
\end{tabular}


Table 1-Continued

\begin{tabular}{|c|c|c|c|c|c|}
\hline $\mathrm{SN}$ & Epochs (days) & Galaxy & $W(6100)(\AA)$ & $W(5750)(\AA)$ & References \\
\hline 1997do BL & 8,21 & UGC 3845 & $\ldots$ & $\ldots$ & Matheson et al. 2008 \\
\hline $1997 \mathrm{dt} \mathrm{CN}$ & $-7,1$ & NGC 7448 & 87 & 14 & Matheson et al. 2008 \\
\hline 1998V CN & 0 & NGC 6627 & 84 & 19 & Matheson et al. 2008 \\
\hline 1998ab SS & $-7,21$ & NGC 4704 & $\ldots$ & $\ldots$ & Matheson et al. 2008 \\
\hline 1998aq CN & $-8,0,7,21,91$ & NGC 3982 & 79 & 16 & Branch et al. 2003 \\
\hline 1998bp CL & 0 & NGC 9495 & 120 & 54 & Matheson et al. 2008 \\
\hline 1998bu CN & $-6,-1,8$ & NGC 3368 & 93 & 21 & $\begin{array}{l}-6,-1: \text { Hernandez et al. } 2000 \\
\text { 8: Jha et al. } 1999\end{array}$ \\
\hline 1998de CL & $-6,0$ & NGC 252 & 164 & 58 & Matheson et al. 2008 \\
\hline 1998dh BL & $-7,0$ & NGC 7541 & 120 & 24 & Matheson et al. (2008 \\
\hline $1998 \mathrm{ec}$ BL & $-1,21$ & NGC 5948 & 124 & 11 & Matheson et al. 2008) \\
\hline 1998 eg CN & $0,6,20$ & NGC 7391 & 95 & 20 & Matheson et al. 2008 \\
\hline 1998es SS & $-7,-1,20,81$ & NGC 3157 & 51 & 7 & Matheson et al. 2008 \\
\hline 1999aa SS & $-11,-7,-1,6,19,81$ & NGC 4469 & 63 & 14 & $\begin{array}{l}-11,6,19,81: \text { Garavini et al. } 2004 \\
-7,-1 \text { : Matheson et al. } 2008\end{array}$ \\
\hline 1999ac SS & $-15,-9,-2,8,86$ & NGC 6063 & 84 & 9 & $\begin{array}{l}-15,-9,-2,8: \text { Garavini et al. } 2005 \\
\text { 86: Matheson et al. } 2008\end{array}$ \\
\hline 1999aw SS & 3 & $\ldots$ & 55 & 2 & Howell \& Nugent 2004 \\
\hline 1999by CL & $-3,7$ & NGC 2841 & 94 & 48 & Garnavich et al. 2004 \\
\hline 1999cc BL & 0,23 & NGC 6038 & 121 & 26 & Matheson et al. 2008 \\
\hline 1999cl BL & $-7,0,8$ & NGC 4501 & 133 & 19 & Matheson et al. 2008 \\
\hline 1999dq SS & $-7,1,6,19$ & NGC 976 & 51 & 10 & Matheson et al. 2008 \\
\hline 1999ee SS & $-7,-2,22$ & IC 5179 & 77 & 9 & Hamuy et al. 2002 \\
\hline
\end{tabular}


Table 1-Continued

\begin{tabular}{|c|c|c|c|c|c|}
\hline $\mathrm{SN}$ & Epochs (days) & Galaxy & $W(6100)(\AA)$ & $W(5750)(\AA)$ & References \\
\hline 1999ej BL & 0 & NGC 495 & 108 & 27 & Matheson et al. 2008 \\
\hline 1999gd BL & 2 & NGC 5472 & 108 & 17 & Matheson et al. 2008 \\
\hline 1999gh BL & 7 & NGC 2986 & $\ldots$ & $\ldots$ & Matheson et al. 2008 \\
\hline 1999gp SS & $-1,7,21$ & $\ldots$ & 52 & 8 & Matheson et al. 2008 \\
\hline 2000B BL & 8,21 & NGC 2320 & $\ldots$ & $\ldots$ & Matheson et al. 2008 \\
\hline 2000E SS & $-6,-2,8$ & NGC 6951 & 78 & 13 & Valentini et al. 2003 \\
\hline 2000cn CL & $-7,21$ & NGC 7043 & $\ldots$ & $\ldots$ & Matheson et al. 2008 \\
\hline 2000cx SS & $2,7,20,89$ & NGC 524 & 49 & 3 & Li et al. 2001a \\
\hline 2000dk CL & 1 & NGC 5217 & 124 & 47 & Matheson et al. 2008 \\
\hline 2000fa CN & 1,21 & NGC 6379 & 96 & 11 & Matheson et al. 2008 \\
\hline 2001V SS & $-13,-7,20$ & NGC 4589 & $\ldots$ & $\ldots$ & Matheson et al. 2008 \\
\hline 2001ay BL & 0 & IC 4423 & 146 & 8 & Howell \& Nugent 2004 \\
\hline 2001el CN & 1,20 & NGC 1448 & 95 & 14 & Wang et al. 2003 \\
\hline 2002bf BL & 3,7 & & 175 & 11 & Leonard et al. 2005 \\
\hline 2002bo BL & $-14,-8,-1,82$ & NGC 3190 & 147 & 13 & Benetti et al. 2004 \\
\hline 2002cx SS & $-1,21$ & $\ldots$ & 26 & 8 & Li et al. 2003 \\
\hline 2002dj BL & $-11,-6,-3,23$ & NGC 5018 & 150 & 14 & Pignata et al. 2008 \\
\hline 2002er BL & $-11,-7,0,6,20$ & UGC 10743 & 129 & 16 & Kotak et al. 2006 \\
\hline $2003 c g \mathrm{CN}$ & $-7,-1,7,23$ & NGC 3169 & 96 & 15 & Elias-Rosa et al. 2006 \\
\hline 2003du CN & $-11,-7,0,7,84$ & UGC 9391 & 85 & 15 & Stanishev et al. 2007a \\
\hline 2003fg SS & 2 & $\ldots$ & 55 & 15 & Howell et al. 2006 \\
\hline $2004 \mathrm{~S} \mathrm{CN}$ & $1,8,19$ & $\ldots$ & 89 & 13 & Krisciunis et al. 2007 \\
\hline $2004 d t$ BL & $-8,-1,20$ & NGC 799 & 186 & 10 & Altavilla et al. 2007 \\
\hline
\end{tabular}


Table 1-Continued

\begin{tabular}{cccccl}
\hline \hline SN & Epochs (days) & Galaxy & $W(6100)(\AA)$ & $W(5750)(\AA)$ & \multicolumn{1}{c}{ References } \\
\hline 2004eo CL & $2,7,21$ & NGC 6928 & 106 & 34 & Pastorello et al. 2007 \\
2005bl CL & $-6,-3,20$ & NGC 4070 & 89 & 39 & Taubenberger et al. 2008 \\
2005cf CN & $-12,-6,0,7$ & $\ldots$ & 97 & 13 & Garavini et al. 2007 \\
& & & & & 83: Wang et al. 2009 \\
2005cg SS & 0,7 & $\ldots$ & 64 & 7 & Quimby et al. 2006 \\
2005hj SS & $-6,0$ & $\ldots$ & 79 & Quimby et al. 2007 \\
2005hk SS & 0,21 & UGC 272 & 29 & 8 & Stanishev et al. 2007b \\
2006X BL & $1,6,98$ & NGC 4321 & 179 & 9 & Wang et al. 2007 \\
2006gz SS & $-14,-2,7$ & IC 1277 & 64 & 12 & Hicken et al. 2007 \\
\hline
\end{tabular}

